\title{
Ciclofotocoagulação com laser diodo em glaucoma refratário, resultado a longo prazo
}

\author{
Cyclophotocoagulation with diode laser in refractory glaucoma, long-term results
}

$\begin{array}{lcccc}\text { Vera } & \text { Christina } & \text { Waller de } & \text { Lima } \\ \text { Paulo } & \text { Augusto } & \text { de Arruda } & \text { Mello } \\ \text { João } & \text { Antonio } & \text { Prata Junior } & \end{array}$

Realizado na Universidade Federal de São Paulo UNIFESP - Sub-Especialidade: Glaucoma

${ }^{1}$ Médica Voluntária do Setor de Glaucoma da Universidade Federal de São Paulo - UNIFESP;

2 Professor Adjunto do Departamento de Oftalmologia da Universidade Federal de São Paulo - UNIFESP e Orientador da Pós-Graduação.

${ }^{3}$ Mestre e Doutor em Oftalmologia pela Universidade Federal de São Paulo - UNIFESP e Chefe do Departamento de Glaucoma da Faculdade de Medicina da Universidade Federal de São Paulo - UNIFESP.

Endereço para correspondência:

End: Rua Ministro Godoy, $860 \mathrm{apt}^{\circ} 3084$ - São Paulo (SP) CEP 05015-000

Recebido para publicação em 06.06 .2000 Aceito para publicação em 23.01.2003

\section{RESUMO}

Objetivo: Relatar experiência de longo prazo com ciclofotocoagulação com laser diodo em glaucomas refratários. Métodos: Foram selecionados casos de glaucomas refratários com indicação de ciclofotocoagulação com laser diodo acoplado a uma sonda G (Iris Medical). Todos os procedimentos foram realizados pelo mesmo cirurgião que realizou 20 aplicações (em média) com $2000 \mathrm{~mW}$ de potência em 270 graus de extensão, com a sonda colocada a 1,5 mm do limbo, por 2,0 a 2,5 s. Foram incluídos somente casos com pelo menos 6 meses de seguimento. Considerou-se sucesso ci-rúrgico Po final $\leq 24 \mathrm{mmHg}$ ou redução maior ou igual a $30 \%$ da Po inicial com remissão dos sintomas. Resultados: 133 casos tiveram acompanhamento médio de 18,1 $\pm 9,6$ meses e observou-se sucesso em $116(87,2 \%)$ casos e $17(12,7 \%)$ casos de insucesso. A Po inicial média foi $48,20 \pm 12,11 \mathrm{mmHg}$ e a final 19,93 $\pm 11,93 \mathrm{mmHg}(\mathrm{p}<0,0001)$, comuma redução média de - 55,62 $\pm 29,37 \%$. As principais complicações foram "phthisis bulbi" em $7(5,2 \%)$ casos e hipotonia prolongada em 6 (4,5\%) casos. Conclusão: Observaramse freqüência de sucesso e níveis de Po pós-operatório satisfatórios, com incidência relativamente baixa de complicações, considerando-se a refratariedade dos casos.

Descritores: Fotocoagulação/métodos; Coagulação por laser/métodos; Esclera; Glauco$\mathrm{ma} /$ cirurgia; Resultado de tratamento

INTRODUÇÃO

Os glaucomas refratários representam grande problema terapêutico tendo em vista que, mesmo com o uso de medicação máxima tolerada, muitos casos mantêm importante sintomatologia. Recursos técnicos como cirurgia fistulisante com antiproliferativos e/ou tubos de drenagem têm sido empregados, com significativas taxas de sucesso ${ }^{(1-3)}$. Entretanto, em algumas situações, esses não logram o controle do glaucoma, fazendo-se necessária a utilização de técnicas ciclodestrutivas ${ }^{(4)}$.

Em 1936 Vogt introduziu a ciclodiatermia e, a seguir, em 1947-50, Bietti introduziu a ciclocrioterapia ${ }^{(5)}$. Desde esta época inúmeras inovações foram introduzidas, até a utilização da energia do laser para destruição dos processos ciliares (ciclofotocoagulação) ${ }^{(4-7)}$.

Os procedimentos ciclodestrutivos visam reduzir a pressão intra-ocular (Po) por diminuição da produção de humor aquoso, através da destruição dos processos ciliares.

A ciclofotocoagulação pode ser realizada por via transescleral, transpupilar ou endoscópica. A via transescleral é a mais referida na literatura e é procedida por técnicas de contato ou não-contato, dependendo se o mecanismo de liberação do laser é colocado ou não em contato com o olho ${ }^{(8-9)}$. 
Os equipamentos mais utilizados têm sido o YAG laser 1064 nm e o laser diodo ${ }^{(10,11)}$.

O laser diodo tem como vantagens: ser portátil e ter um menor preço que os outros laseres utilizados para o mesmo fim, como o ND Yag laser ${ }^{(11-12)}$.

Com relação ao laser diodo, o tratamento é realizado pela técnica transcleral de contato. Estudos referem que o tratamento foi bem sucedido em 14 pacientes portadores de glaucomas refratários, obtendo valores da Po abaixo de $21 \mathrm{mmHg}^{(13)}$. Após um ano de tratamento, alguns autores referem taxas de sucesso de $52 \%{ }^{(14)}$.

Devido à alta incidência de complicações, como irite, dor, hemorragia, hipotonia e "phthisis bulbi", recomenda-se que os pacientes sejam cuidadosamente selecionados e que apresentem acuidade visual baixa e sem possibilidade de submeter-se a outros tipos de terapia ${ }^{(1,12,15)}$.

\section{MÉTODOS}

Antes do início do estudo, o protocolo foi submetido à apreciação da Comissão de Ética da Escola Paulista de Medicina, tendo obtido a sua aprovação. Foram selecionados portadores de glaucoma refratário em tratamento no Setor de Glaucoma da UNIFESP - Escola Paulista de Medicina. Considerouse como glaucoma refratário aquele que apresentava níveis de Po incompatíveis com a preservação da função visual e/ou que estivesse associado a severa sintomatologia, em indivíduos sob máxima medicação tolerada e sem indicação para outras técnicas antiglaucomatosas, seja por seguidos insucessos ou por falta de condição anatômica ou clínica para sua realização.

Não foram incluídos no estudo pacientes com acuidade visual melhor que 20/100 e os glaucomas absolutos sem sintomatologia clínica. Os casos com menos de seis meses de acompanhamento pós-operatório não foram considerados no estudo, a não ser que ocorresse insucesso do procedimento neste período.

Considerou-se hipotonia como pressão final inferior a $5 \mathrm{mmHg}$.

Os procedimentos foram realizados em bases ambulatoriais após anestesia retrobulbar com lidocaína $2 \%$ e bupivacaína $0,5 \%$, na proporção de $60 \%$ e $40 \%$, respectivamente. Estando o paciente em decúbito dorsal, a sonda do aparelho foi posicionada a $1,5 \mathrm{~mm}$ do limbo e 16 a 21 disparos foram realizados em 270 graus, com potência de 1500 a $2000 \mathrm{~mW}$ e duração de 2 a 2,5 s. O equipamento utilizado foi o Laser Diodo Oculight SL fornecido pela Iris Medical, de comprimento de onda $810 \mathrm{~nm}$ e área de $400 \mu \mathrm{m}$ de diâmetro, acoplado à sonda, para liberação do laser tipo G Probe (Iris Medical). Após o procedimento, todos os casos receberam antiinflamatório injetável não esteróide para controle da dor e foram prescritos colírios de antibiótico e corticosteróide (Maxitrol ${ }^{\circledR}$ - Alcon) e atropina (Atropina $^{\circledR}$ - Frumtost), por períodos variáveis, dependendo da intensidade do processo inflamatório subseqüente. A me- dicação antiglaucomatosa foi suspensa gradativamente após o procedimento.

Para efeito de estudo, no pré-operatório, foram anotados o tipo de glaucoma, medicação utilizada, nível da Po e acuidade visual. No pós-operatório a Po foi medida imediatamente após o tratamento e no acompanhamento de 6, 12, 18, 24, 30, 36 meses. Considerou-se como acuidade visual final a obtida no último retorno pós-operatório disponível.

Considerou-se sucesso cirúrgico valores de $5 \mathrm{mmHg} \geq \mathrm{Po}$ final $\leq 24 \mathrm{mmHg}$ com ou sem medicação antiglaucomatosa e/ou redução percentual da Po final maior ou igual a $30 \%$, com redução significativa da sintomatologia. Perda de acuidade visual foi considerada quando ocorreu diminuição da acuidade visual de 20/100 para 20/300, 20/300 para conta dedos, conta dedos para projeção luminosa, projeção luminosa para percepção luminosa e perda da percepção pré-tratamento.

A análise estatística comparou a Po inicial (pré-tratamento) pelo teste de Friedman com os valores obtidos após 6,12 , $18,24,30$ e 36 meses, e para a diferença entre o valor inicial e final (última visita) pelo teste de Wilcoxon. Calculou-se também os valores da redução percentual da Po em relação a inicial. O teste de Friedman revelou diferença estatisticamente significante entre as medidas. A fim de determinar onde estão as diferenças significativas, realizamos o teste de Dunn que nos fornece uma comparação entre pares de variáveis.

\section{RESULTADOS}

No estudo foram incluídos 133 pacientes, com média de idade de 50 anos, com tempo de seguimento de 36 meses com média de $18,1 \pm 9,6$ meses.

Incluímos neste estudo $50(37 \%)$ pacientes por apresentarem glaucoma neovascular, $30(23 \%)$ com glaucoma secundário, 46 (34\%) com glaucoma absoluto, 2 (1,5\%) com glaucoma maligno, $5(3,7 \%)$ com glaucoma congênito.

Após acompanhamento observou-se que $116(87,2 \%)$ dos pacientes foram considerados sucesso, e 17 (12,7\%) foram considerados insucesso, pois 4 (3\%) apresentaram Po elevada pós-procedimento e $13(9,7 \%)$ pacientes apresentaram Po inferior a $5 \mathrm{mmHg}$.

Devemos considerar que após 36 meses ( 3 anos) apenas 25 (18,75\%) concluíram o estudo; em 2 anos obtivemos apenas $36,75 \%$ de adesão ao seguimento.

A tabela I e gráfico I apresentam valores médios de Po nos períodos estudados e o percentual de queda da Po nos períodos.

A análise estatística mostrou que os valores de Po diferiram entre si de forma significante $(\mathrm{p}<0,0001)$. O teste de comparações múltiplas detectou que a Po final foi estatisticamente inferior a todas as outras medidas e não foi observada diferença entre Po de 6 e 36 meses.

A análise da variação percentual de Po pós-operatória detectou que os valores diferiram estatisticamente entre si. Observou-se que os valores obtidos no pré e pós-operatório 


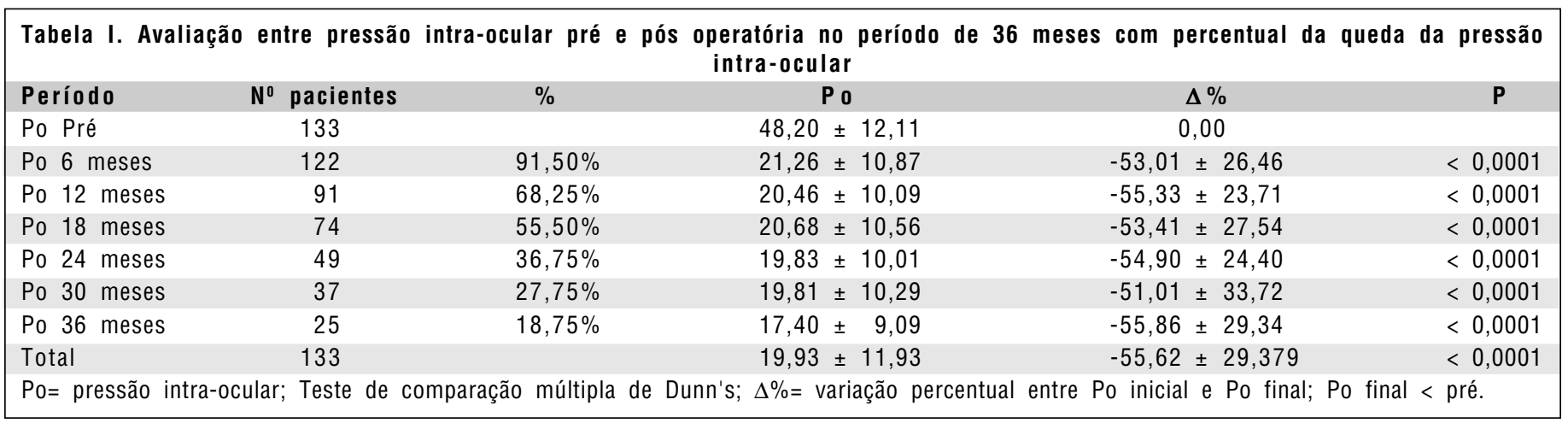

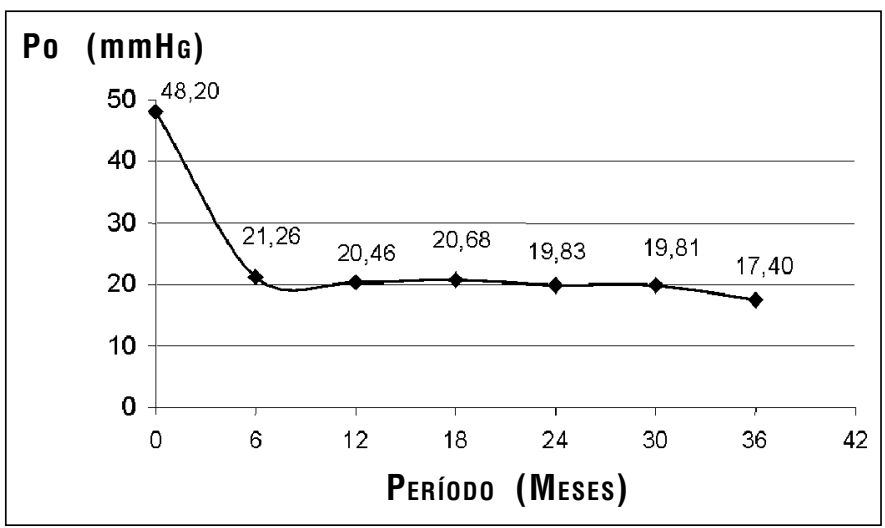

Gráfico I - Estudo comparativo de Po x Período 'pós diodo da queda da Po

foram estatisticamente diferentes; na variação entre 6, 12, 24, 30 e 36 meses, não houve diferença estatisticamente significante $(\mathrm{p}>0,05)$ entre eles.

$O$ resultado da análise de sobrevivência de Kaplan-Meier encontra-se no gráfico II.

As complicações observadas foram: hemorragia vítrea, 1 caso $(0,75 \%)$; hifema, 4 casos $(3 \%)$; hipotonia, 6 casos $(4,5 \%)$, "phthisis bulbi", 7 casos $(5,2 \%)$, mas como decorrência de

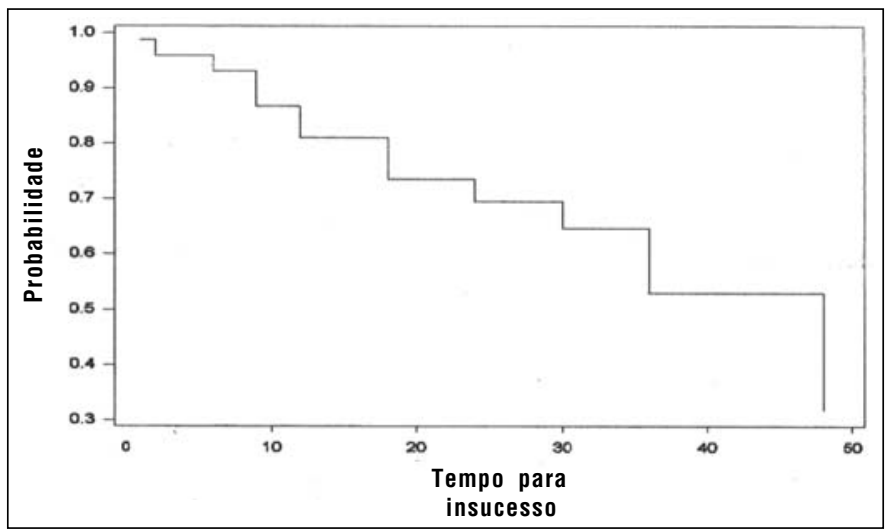

Gráfico II - Análise de sobrevivência de Kaplan-Meier probabilidade de insucesso do controle de Po pós diodo laser utilizando como critério Po final $\leq 24 \mathrm{~mm}$ Po final $\leq 30 \%$ Po inicial uma reaplicação com os mesmos parâmetros utilizados na aplicação inicial.

Baixa de acuidade visual foi observada em 18 casos $(13,5 \%)$, no entanto constatamos melhora da acuidade visual em 6 casos $(4,5 \%)$ o restante permaneceu com a mesma acuidade visual inicial.

Em relação à quantidade de medicação, apenas uma medicação é utilizada em 67 casos (50,3\%); duas medicações em 12 casos $(9,0 \%)$; e o restante 41 casos $(30,8 \%)$ encontram-se sem medicação hipotensora.

Para obtermos uma liberação lenta com baixa energia, aumentamos o tempo de aplicação $(2,0$ - 2,5), aumentando a temperatura do tecido, sem ultrapassar o ponto de coagulação, o que ocorreria se fosse utilizada uma liberação mais rápida $^{(11)}$.

\section{DISCUSSÃO}

Temos verificado a eficiência da ciclofotocoagulação com laser diodo transescleral após seguimento de 36 meses, apesar de termos $18,75 \%$ de adesão neste período com pequena taxa de complicações e com redução do número de medicação uti$\operatorname{lizada}^{(14,17)}$.

Neste estudo observou-se uma taxa de sucesso de 116 olhos (87,2\%) com Po de final de 19,93 $\pm 11,93$, corroborando os resultados encontrados na literatura, e insucesso de 4 casos $(3,0 \%)$ com aumento da Po no seguimento pós-operatório, se bem que nestes casos tivemos a retirada da medicação oral (acetazolamida) e diminuição da sintomatologia inicial significativa.

Apesar de em 122 pacientes conseguirmos um seguimento de 6 meses, em apenas 25 pacientes termos um seguimento de 36 meses acreditamos que este tratamento é eficaz e os resultados não se alterariam se tivéssemos maior número de pacientes no seguimento, como foi observado na literatura. Salientamos que este é um estudo que deve ser mantido por mais tempo.

A análise de sobrevivência de Kaplan-Meier nos permite analisar que até 3 anos, aproximadamente 53\% dos procedimentos foram considerados sucesso.

Sem dúvida, os resultados de sucesso decresceriam signi- 
ficativamente se fossem utilizados critérios de sucesso semelhante ao empregado para análise da cirurgia fistulisante (Po $\leq$ $21 \mathrm{mmHg}$ ). Entretanto, o critério adotado nesta série veio refletir o objetivo do procedimento, qual seja o de aliviar a sintomatologia e diminuir a quantidade de medicação utilizada, principalmente nos casos de glaucoma absoluto e neovascular.

Contudo quando comparamos com valores encontrados na literatura relativo à baixa de acuidade visual após ciclocrioterapia entre 5 a $69 \%$; hipotonia ou "phthisis bulbi" entre 3 a $17 \%$ constatamos a eficiência do procedimento ${ }^{(18)}$.

Considerando a gravidade dos casos tratados, baixos índices de complicações foram obtidos e, como esperado, casos de "phthisis" foram observados ${ }^{(15)}$.

Em casos de reaplicação, utilizamos menor número e menor energia que a utilizada inicialmente, para diminuirmos a incidência de complicações ${ }^{(17)}$.

$\mathrm{O}$ resultado indica que a ciclofotocoagulação com laser diodo pode ser uma alternativa para o tratamento de glaucoma refratário, desde que haja criteriosa seleção de pacientes, tendo em vista que consiste em um tratamento de alta eficiência, baixo custo e, sendo procedimento extra-ocular, pode ser realizado ambulatorialmente com segurança e facilidade com baixo grau de complicação.

Quando comparada com a ciclocrioterapia, que é um método mais tradicional, verificamos que esta apresenta maior incidência de complicações como "phthisis bulbi" e maior reação inflamatória pós-operatória e muitas vezes necessita maior número de reaplicações para obtenção da melhora do quadro clínico do paciente. Já a endociclofotocoagulação é um procedimento muito mais complexos necessitando maior habilidade do cirurgião e equipamento muito mais sofisticado ${ }^{(19-21)}$.

\section{ABS TRACT}

Purpose: To report long-term experience with cyclophotocoagulation using diode laser in refractory glaucomas. Methods: We selected cases of refractory glaucoma with indication for cyclophotocoagulation (CPC) with diode laser using a $\mathrm{G}$ probe (Iris Medical). All procedures were performed by the same surgeon: twenty applications of $2000 \mathrm{~mW}$ power at 270degree extension. The probe placed set at $1.5 \mathrm{~mm}$ from the limbus during 2.0 a $2.5 \mathrm{~s}$. Only cases with at least 6 months of follow-up were included. The procedure was considered successful when IOP was $\leq 24 \mathrm{mmHg}$ or decrease was larger or equal to $30 \%$ of the initial IOP with remission of symptoms. Only 25 patients (18.75\%) concluded the study (36 months). Results: One hundred and thirty cases had a mean follow-up of $18,1 \pm 9,6$ months with $116(87.2 \%)$ successful cases and 17 $(12,7 \%)$ insuccess ful cases. Initial and final IOP were, $48.20 \pm$ $12.11 \mathrm{mmHg}$ and $19.93 \pm 11,93 \mathrm{mmHg}$ respectively $(\mathrm{p}<0.0001)$. Main complications were: "Phthisis bulbi" in 7 (5.2\%) cases and long-term hypotony in $6(4.5 \%)$ cases. Conclusions: Considering the severity of our cases, we conclude that the long-term results of cyclophotocoagulation with diode laser for refractory glaucomas were satisfactory, with an acceptable incidence of complications.

Keywords: Photocoagulation/methods; Light coagulation/ methods; Sclera; Glaucoma/surgery; Treatment outcome

\section{REFERENCIAS}

1. Omi CA Almeida GV, Cohen R, Mandia Júnior C, Kwitko S. Modificações da técnica de Schocket em 55 olhos portadores de glaucoma refratários primeiros resultados [Abstract]. Arq Bras Oftalmol 1989;52:134.

2. Ayyala RS, Pieroth L, Vinals AF, Godstein MH, Shuman JS, Netland PA, et al. Comparison of mitomycin $\mathrm{C}$ trabeculectomy glaucoma drainage device implantation, and laser neodymium: YAG ciclophotocoagulation in the management of intractable glaucoma after penetrating keratoplasty. Ophthalmology 1998;105:1550-6.

3. Eid TE, Katz LJ, Spaeth GL, Augsburger JJ. Tube-shunt surgery versus neodymiun-YAG cyclophotocoagulation in the management of neovascular glaucoma. Ophthalmology 1997; 104:1692-700.

4. Beckman H, Waeltermann J. Transscleral ruby laser cyclocoagulation. Am J Ophthalmol 1984;98:88-95.

5. Bietti G. Surgical Interventions on the ciliary body: new trends for the relief of glaucoma JAMA 1950;142:889-96.

6. Bellows AR, Grant WM. Cyclocryotherapy of chronic open-angle glaucoma in aphakic eyes. Am J Ophthalmol 1978;85:615-21

7. Beckman H. Transscleral laser cyclocoagulation. Trans New Orleans Acad Ophthalmol 1985;33:148-53.

8. Walland MJ. Diode laser cyclophotocoagulation: dose standardized therapy in end stage glaucoma. Aust N Z J Ophthalmol 1998;26:135-9.

9. Pressner PR, Boos N, Fassbender K, Schwenn O, Pfeiffer N. Real-time control for transscleral cyclophotocoagulation Graefes Arch Clin Exp Ophthalmol 1997;235:794-801.

10. Yap-Veloso MI, Simmons RB, Echelman DA, Gonzales TK, Vieira WJ, Simmons RJ. Intraocular pressure control after contact transscleral diode cyclophotocoagulation in eyes with intractable glaucoma. J Glaucoma 1998;7:319-28.

11. Klein S, Rockwood EJ, Baerveldt G. Diode laser versus contact transscleral Nd: Yag Cyclophotocoagulation (CPC) for incontrolled Glaucoma, ARVO. Invest Ophthalmol Vis Sci 1996;37:1192.

12. Youn J, Cox TA, Herndon LW, Allinghan RR, Shields MB. A clinical comparison of transscleral cyclophotocoagulation with neodymiun: YAG and semiconductor diode lasers. Am J Ophthalmol 1998;126:640-7.

13. Hennis HL, Stewart WC. Semiconductor diode laser transscleral cyclophotocoagulation in patients with glaucoma. Am J Ophthalmol 1992;113:81-5.

14. Brancato R, Trabucchi G, Bettin P, Fiori M. One-year follow up study of contact transscleral cyclophothocoagulation with diode laser, ARVO, Invest Ophthalmol Vis Sci 1993;34(4 suppl):175.

15. Dickens CJ, Ngueyen N, Mora JR, Iwack AG, Gaffney MM, Wong PC, Tran $\mathrm{H}$. Long term results of noncontact transcleral neodymium. Yag cyclophotocoagulation. Ophthalmology 102:1777-81.

16. Fankhauser F, Kwasniewskas S. Initial ciliary ablation with TSCPC Ophthalmology 1994;104:568-70.

17. Bloom PA, Tsai JC, Sharma K, Miller MH, Rice NS, Hitchings RA, Khaw PT. "Cyclodiode". Trans-scleral diode laser cyclophotocoagulation in the treatment of advanced refractory glaucoma. Ophthalmology 1997;104:1508-20.

18. Mastrobattista JM, Luntz M. Ciliary body ablation: where are we and how did we get here? Surv Ophthalmol 1996;41:193-213.

19. Lima FE, Avila M, Ribeiro C. Ciclofotocoagulação transescleral com laser diodo em glaucoma neovascular. Rev Bras Oftalmol 1996;55:627-33.

20. Brancato R, Carassa RG, Bettin P, Fiori M, Trabucchi G. Contact transscleral cyclophotocoagulation with diode laser in refractory glaucoma. Eur $\mathrm{J}$ Ophthalmol 1995;5:32-9.

21. Tsai JC, Bloom PA, Franks WA, Khaw PT. Combined transscleral diode laser cyclophotocoagulation and transscleral retinal photocoagulation for refractory neovascular glaucoma. Retina 1996;16:164-6. 